\title{
Plaster technique for filling up a future entry at the suture hole in type A aortic dissection
}

\author{
Shinichi Ishida ${ }^{1}$, Masato Mutsuga ${ }^{2}$, Takashi Fujita ${ }^{1}$, and Kei Yagami ${ }^{1}$ \\ ${ }^{1}$ Gifu Kenritsu Tajimi Byoin \\ ${ }^{2}$ Nagoya University Graduate School of Medicine Faculty of Medicine
}

July 1, 2021

\begin{abstract}
Although the surgical technique for acute type A aortic dissection markedly improved in the last decade, perioperative mortality and morbidity rates remain dramatically high. Therefore, we introduce the novel "plaster technique" using the single interrupted

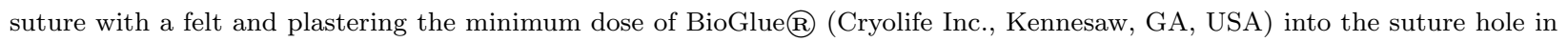
this report. We found that the plaster technique using a felt pledget and minimum dose of BioGlue is effective for fragile aortic walls, as in patients undergoing acute aortic dissection and is a simple, safe, and durable technique to strengthen the suture line.
\end{abstract}

Title: Plaster technique for filling up a future entry at the suture hole in type A aortic dissection Authors:

Shinichi Ishida $\mathrm{MD}^{1}$, Masato Mutsuga MD, $\mathrm{PhD}^{2}$, Takashi Fujita $\mathrm{MD}^{1}$, Kei Yagami MD, $\mathrm{PhD}^{1}$

${ }^{1}$ Department of Cardiac Surgery, Gifu Prefectural Tajimi Hospital, Tajimi, Gifu, Japan.

${ }^{2}$ Department of Cardiac Surgery, Nagoya University Graduate School of Medicine, Nagoya, Aichi, Japan.

Short running title: Plaster technique

Keywords: aorta and great vessels, type A aortic dissection, suture hole, future entry

Acknowledgement: There are no sources of funding for this manuscript.

Conflicts of Interest: The authors declare that there are no conflict of interests.

Corresponding Author:

Shinichi Ishida, 5-161 Maebata-cho, Tajimi-city, Gifu 507-8522, Japan, Tel: +81-572-22-5311, email:shin1dinho@yahoo.co.jp

Abstract:

Although the surgical technique for acute type A aortic dissection markedly improved in the last decade, perioperative mortality and morbidity rates remain dramatically high. Therefore, we introduce the novel "plaster technique" using the single interrupted suture with a felt and plastering the minimum dose of

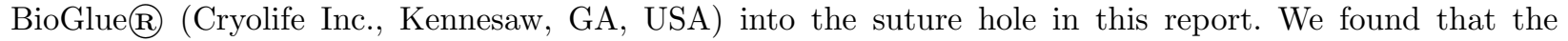
plaster technique using a felt pledget and minimum dose of BioGlue is effective for fragile aortic walls, as in patients undergoing acute aortic dissection and is a simple, safe, and durable technique to strengthen the suture line. 


\section{Introduction:}

The surgical technique for acute type A aortic dissection (AAAD) markedly improved in the last decade; however, perioperative mortality and morbidity rates remain dramatically high. ${ }^{1}$ Furthermore, the aortic wall of patients who underwent aortic dissection is fragile, and reoperation may cause new entry or aneurysmal formation at the anastomotic site. ${ }^{2}$ A small needle hole cracking into the intimal layer is rarely reported, owing to the aortic wall vulnerability even if it remains intact. Thus, performing re-anastomosis seems difficult using just one reserved single needle hole, which might induce the occurrence of future suture entry and reoperation. We believe that filling up this hole with a simple and short-time technique is needed. Therefore, we introduce the novel "plaster technique" using the single interrupted suture with a felt and plastering the minimum dose of BioGlue@ (Cryolife Inc., Kennesaw, GA, USA) into the suture hole. Institutional review board approval was exempted at our institution for this retrospectively designed report.

Surgical Technique:

The surgical procedure for AAAD is usually performed through median sternotomy, and cardiopulmonary bypass is established via femoral artery cannulation and bicaval drainage and left ventricular venting. Systemic core cooling is initiated until the nasopharyngeal temperature reaches $22^{\circ} \mathrm{C}$. The ascending aorta is gently clamped and cardiac arrest is achieved by a retrograde cardioplegia.

The proximal side of the dissected aorta is opened by a low transverse incision circumferentially. After resecting the dissected ascending aorta, its proximal side is created by combining intimal and adventitial layers by injecting a small dose of BioGlue. The felt strip is placed outside the aorta, and the turn-upped prosthetic graft is inserted into the lumen. The four everting mattress stitches with 4-0 polypropylene are placed to fix the aortic wall and the prosthetic graft at $90^{\circ}$ intervals, and running sutures are performed by keeping the same depth of the suture to complete the anastomosis.

After the anastomosis, the suture line is confirmed inside to examine the presence of any new intimal tear caused by the suture and, additionally, to identify the part of fragile intima even those without tears. To fix this new tear at a suture hole, a double-arm 4-0 polypropylene suture with a felt pledget is inserted from inside the aortic lumen to outside the graft. A minimum dose of BioGlue is plastered just above the suture hole, and the suture with a felt pledget is tied gently and covered the hole completely (Figure 1). The video provides an overview of this surgical technique (Video 1).

When the nasopharyngeal temperature reaches $22^{\circ} \mathrm{C}$, circulatory arrest is initiated and the aorta is declamped. After checking for intimal layer tears, the dissected aortic wall is resected including the tear. Additionally, the aortic wall is trimmed for the distal-side anastomosis in the same manner. The distal-side anastomosis is performed as in the proximal side. After completing the distal anastomosis, body reperfusion is initiated from the graft branch. Finally, proximal and distal grafts are anastomosed with 2-0 polyester running sutures. Postoperative computed tomography scan shows complete repair at the proximal suture site performed using the plaster technique (Figure 2) to strengthen a suture hole by plastering the wall and to prevent the blood stream into the suture entry.

Discussion:

Various methods have been used for AAAD, with ascending aorta replacement as the most common approach, with aortic valve and aortic root repair or replacement and aortic arch replacement as necessary. Basically, resecting the intimal tear and preventing the antegrade flow into the false lumen are important. ${ }^{3}$ The new entry from the suture hole at the anastomotic site could cause false lumen enlargement and aneurysm and should be closed intraoperatively. The present plaster technique is a new method used to fill up a new entry caused by a suture hole. A felt pledget is used to compensate the dissected wall vulnerability; furthermore, BioGlue is plastered to perform strong fixation. Minimum dose of BioGlue should be used to prevent falling off and embolus formation. With regard to the pledget, the autologous myocardium patch can be substituted by or combined with the felt.

This technique was performed in six patients with AAAD with a new suture hole at the anastomotic site. 
The maximum follow-up period was 18 months, and no patients had new entry or aneurysmal formation at the suture line postoperatively.

Conclusion:

The plaster technique using a felt pledget and minimum dose of BioGlue is effective for fragile aortic walls, as in patients undergoing acute aortic dissection and is a simple, safe, and durable technique to strengthen the suture line.

Author contributions:

S.I., M.M., T.F. and K.Y. designed and performed the experiments, analyzed data and interpreted it. S.I. and K.Y. Drafted article. S.I., M.M., F.T. and K.Y. revised it critically. S.I., M.M., F.T. and K.Y. approved of the article, collected data and supported technical and logistical.

\section{References:}

1. Geirsson A, Ahlsson A, Franco-Cereceda A, et al. Hospital volumes and later year of operation correlates with better outcomes in acute Type A aortic dissection. Eur J Cardiothorac Surg . 2018;53(1):276-281.

2. Tamura K, Chikazawa G, Hiraoka A, Totsugawa T, Sakaguchi T, Yoshitaka H. The prognostic impact of distal anastomotic new entry after acute type I aortic dissection repair. Eur $J$ Cardiothorac Surg. $2017 ; 52(5): 867-873$.

3. Matalanis G, Ip S. A new paradigm in the management of acute type A aortic dissection: Total aortic repair. J Thorac Cardiovasc Surg.2019;157(1):3-11.

Figure legends:

Figure 1.

A plaster technique schema. In the image above, checking the suture line from inside whether a new intimal tear caused by the suture hole is present and, additionally, to identify the part of fragile intima even if no tear occurred. In the image below, passing a double-arm 4-0 polypropylene suture with a felt pledget from inside the aortic lumen to outside the graft. Injecting BioGlue minimally between the pledget and intimal layer. Tying suture gently and fitting the pledget and aortic wall to plaster the tear.

Figure 2.

Postoperative computed tomography scan shows the complete repair at the proximal suture site performed the plaster technique.

Supplementary material:

Video 1.

The plaster technique for the proximal side anastomosis during the ascending aortic dissection. After the anastomosis, the suture line is confirmed from the inside to examine whether a new intimal tear caused by the suture is present and, additionally, to identify the part of fragile intima even if no tear occurred. To fix the new intimal tear, double-arm 4-0 polypropylene suture with the pledget is passed from inside the aortic lumen to outside the graft. In this case, the felt and autologous myocardium are used together. BioGlue is injected minimally between the pledget and intimal layer and tied suture gently. This technique can strengthen the suture line such as the plaster. 

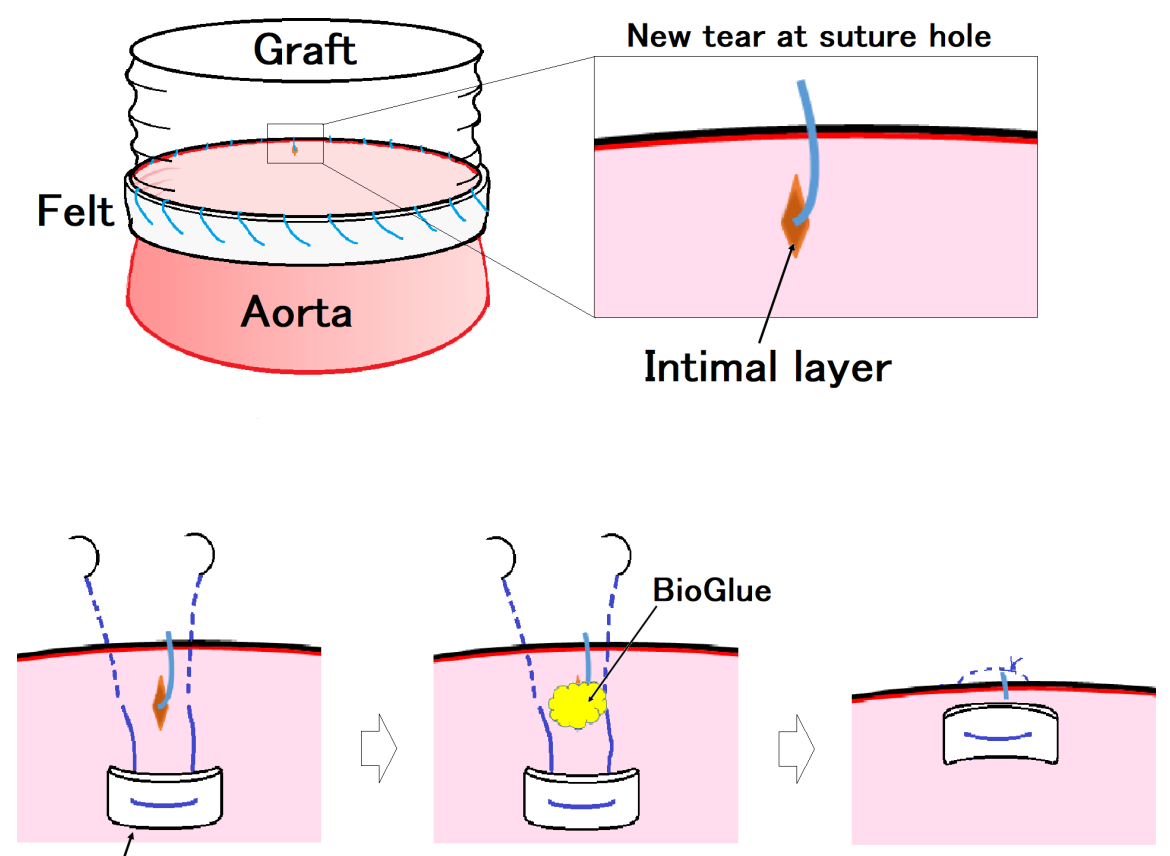

Felt pledget
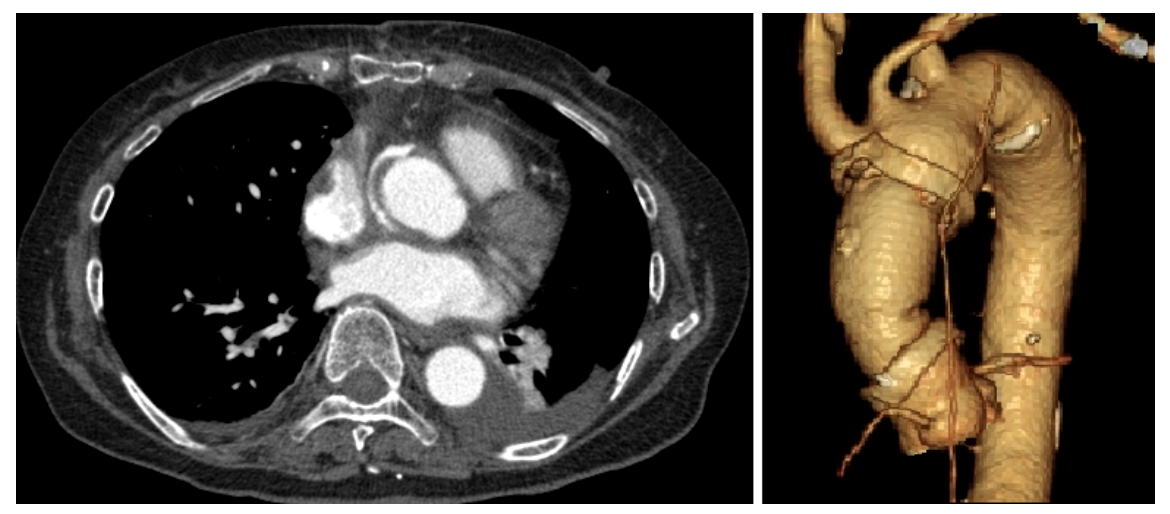